\title{
SPECIAL AVERAGING METHOD FOR SOLVING SOME 3-D HEAT TRANSFER PROBLEMS
}

\author{
Aivars Aboltins ${ }^{1}$, Ilmars Kangro ${ }^{2}$, Harijs Kalis ${ }^{3}$ \\ ${ }^{1}$ Latvia University of Life Sciences and Technologies, Latvia; \\ ${ }^{2}$ Rezekne Academy of Technologies, Latvia; \\ ${ }^{3}$ Institute of Mathematics and Computer Science, University of Latvia, Latvia \\ aivars.aboltins@1lu.lv, ilmars.kangro@rta.lv, harijs.kalis@lu.lv
}

\begin{abstract}
We consider the special conservative averaging method for solving the heat transfer boundary-value problem in the 3-D domain. Looking back to the history of mathematics, integral parabolic splines related to the conservative averaging method (CAM), in 1980s A. Buikis developed CAM for partial differential equations with discontinuous coefficients in environments with a layered structure. We consider newly designed for CAM the special integral hyperbolic type splines for solving some 3-D heat transfer initial-boundary value problems. Using these types of splines, the present problem is reduced to the 2-D problem with respect to one coordinate. This procedure also allows reducing the 2-D problem to 1-D initial-boundary value problem and to initial value problem for ordinary differential equations. Thus, the solution to the problem can be obtained analytically. The solution of corresponding 3-D initial-boundary value problem is obtained also numerically, using for approach differential equations of discretization in space applying the differences. This method can be considered as a generalization of the method of finite volumes, because it can solve BVP with convection, not only in z-direction, but also in ydirection and $\mathrm{x}$-direction, based on the CAM, and, at the same time, through transformation, moving to the obtained BVP without convective terms. The approximation of the 3-D nonstationary problem is based on the implicit finite-difference and alternating direction (ADI) methods. The numerical solution is compared with the spline solution. The publication provides a wide-ranging insight into the practical uses of 3-D heat transfer boundaryvalue problems in general, as well as the effectiveness of the CAM application, in conjunction with the ADI method, is analyzed.
\end{abstract}

Keywords: 3-D problem, PDE, heat transfer, averaging method, splines, analytical solution.

\section{Introduction}

During the modelling of thermal systems we have to, due to their complexity, work with a number of simplifications. In practice, however, these simplifications have no profound effect on the model's accuracy. During the creation of regulation we have to account for the slow dynamic of the system, determine which parameters affect individual heat flows and adjust accordingly.

Heat transfer problems and their mathematical modelling are well known. As the need for a more precise description of the process, a more accurate mathematical model increases the difficulty of solving this mathematical problem. Many real convective heat transfer problems have been described mathematically and an analysis of their numerical results has been given [1]. Assumptions are often used to solve these problems, which simplify the problem itself and thus the solution.

On the other hand, using a mathematical apparatus, it is possible to simplify the mathematical description of the problems under consideration and solve them with simplified, well-known methods, and to evaluate the accuracy of these solutions. This is especially true for solving heat transfer problems in multilayer environments under changing process conditions [2;3]. Another area of widespread concern is the 3-D environment. For the most part, problems are considered in 1-D, 2-D environments or symmetric environments, where it is easier to find a solution. Boiling heat transfer during fluid flow is analyzed at [4]. Two dimensional temperature fields are determined at this case. 3-D heat transfer through cylindrical structures (filled with oil) with irregular cross-sections, which are bounded by a homogeneous elastic medium and heated, is described in [5]. Here 3-D problem can be computed as a summation of 2-D solutions. The natural-convection thermal performance of a dielectric liquid in a cubical module triggered by a varying rectangular heat generation source is numerically examined at [6].Researchers use programming packages very widely. 3-D case of natural convection inside a rectangular enclosure with a discretely heated sidewall has been investigated numerically and experimentally with ANSYS in [7]. The mathematical model for liquid film flow in 3-D formulation has been developed with consideration of thickness changes in vertical and horizontal directions [8].

3-D convection-diffusion problem is considerd in [9]. It appears that the solution to the problem requires substantial mathematical collateral resources, both in theoretical terms (fourth-order alternating 
direction implicit scheme) and in terms of the use of numerical calculation algorithms (threedimensional convection-diffusion equation). Metal distribution in peat layer blocks is modelled in [10].

The task of sufficient accuracy numerical simulation of quick solution of 3-D problems for mathematical physics is important in known areas of the applied sciences. To achieve this goal for engineering calculation the conservative averaging method (CAM) is chosen by using special integral type splines. The application of this method in modelling the combustion process is shown [11].

It is a necessity to solve the 3-D initial-boundary-value problems for parabolic type partial differential equations of second order with constant parameters. The special spline which interpolates middle integral values of a piece-wise smooth function is defined. A. Buikis considered CAM with parabolic type splines for mathematical simulation of the mass transfer process in multilayered underground systems [12]. The theoretical basis of the special parabolic, hyperbolic and exponential type splines with their practical applications has been developed in [13-15].

The conservative averaging method has been applied in the technical sphere, modelling the heat distribution in the 3-D area of automotive fuse [16]. A cylindrical mathematical model of automotive fuse to characterize the heat-up process in the fuse is described by partial differential equations of transient heat conduction. CAM with integral parabolic type splines has been used to get the approximated solution of studied problem with analytical formulas [17]. The conservative averagingreconstruction method (Ring Average) is used for MHD solvers applying explicit finite-volume methods for solving MHD flow problems in cylindrical/spherical geometries. Here, the volume-averaged conservative fluid variables (mass, momentum, energy) are usually defined at cell centres. Thus, the averaging-reconstruction of the fluid variables is essentially a one-dimensional process along the azimuthal direction, and therefore the conservative fluid variables in each ring have been reconstructed by the piecewise parabolic method [18]. Here CAM for special hyperbolic type splines is developed. With the help of these splines the 3-D initial-boundary value (IBV) of the heat transfer problem with respect to one coordinate is reduced to 2-D and 1-D IBV problems. Unlike previous investigations, here we use transformation in PDEs for reducing the convection-diffusion heat transfer problem to the problem without convection terms. The solution of the corresponding 3-D initial-boundary value problem is obtained also numerically, using the implicit finite-difference and alternating direction (ADI) methods of J. Douglas and H. H. Rachford [19].

These splines in every direction of averaging contain parameters, where it can be chosen so that the error of the solution is decreasing. In the limit case, when the spline parameters tend to zero, we get the integral parabolic spline, obtained from A. Buikis [12].

\section{Mathematical model}

The nonstationary convection-diffusion process of heat transfer is considered in 3-D parallelepiped

$$
\Omega=\left\{(x, y, z): 0 \leq x \leq L_{x}, 0 \leq y \leq L_{y}, 0 \leq z \leq L_{z}\right\} .
$$

We will find the distribution of the temperature $u=u(x, y, z, t),{ }^{\circ} \mathrm{C}$ at the point $(x, y, z, t) \in \Omega$ and at the time $t$ from the following 3-D initial-boundary value problem (IBVP) for partial differential equation (PDE) with convection in $z$ direction: 


$$
\left\{\begin{array}{l}
\frac{\partial u}{\partial t}=\frac{\partial}{\partial x}\left(D_{x} \frac{\partial u}{\partial x}\right)+\frac{\partial}{\partial y}\left(D_{y} \frac{\partial u}{\partial y}\right)+\frac{\partial}{\partial z}\left(D_{z} \frac{\partial u}{\partial z}\right)+r_{z} \frac{\partial u}{\partial z}, \\
x \in\left(0, L_{x}\right), y \in\left(0, L_{y}\right), z \in\left(0, L_{z}\right), t \in\left(0, t_{f}\right) \\
\frac{\partial u(0, y, z, t)}{\partial x}=\frac{\partial u(x, 0, z, t)}{\partial y}=\frac{\partial u(x, y, 0, t)}{\partial z}=0 \\
D_{x} \frac{\partial u\left(L_{x}, y, z, t\right)}{\partial x}+\alpha_{x}\left(u\left(L_{x}, y, z, t\right)-u_{a x}\right)=0 \\
D_{y} \frac{\partial u\left(x, L_{y}, z, t\right)}{\partial y}+\alpha_{y}\left(u\left(x, L_{y}, z, t\right)-u_{a y}\right)=0 \\
D_{z} \frac{\partial u\left(x, y, L_{z}, t\right)}{\partial z}+\alpha_{z}\left(u\left(x, y, L_{z}, t\right)-u_{a z}\right)=0, u(x, y, z, 0)=u 0(x, y, z),
\end{array}\right.
$$

where $D_{x}, D_{y}, D_{z}-$ constant heat diffusion coefficients, $\mathrm{m}^{2} \cdot \mathrm{s}^{-1}$;

$\alpha_{x}, \alpha_{y}, \alpha_{z}-$ constant heat transfer coefficients in the 3 kind boundary conditions, $\mathrm{m} \cdot \mathrm{s}^{-1}$;

$u_{a z}, u_{a y}, u_{a x}-$ are the given temperatures on the boundaries, ${ }^{\circ} \mathrm{C}$;

$r_{z}-$ constant convective velocity in the $\mathrm{z}$-direction, $\mathrm{m} \cdot \mathrm{s}^{-1}$;

$t_{f}$ - final time, $\mathrm{s}$;

$u 0(x, y, z)$ - given initial temperature, ${ }^{\circ} \mathrm{C}$.

It should be said that themes of tasks/problems solved by PDE (also the above mentioned convection-diffusion IBVP (1)) include different, often very distinguished areas of science and technique, such as economics, fluid dynamics, forecasting, astrophysics, oceanography, meteorology, etc., [20; 21].

Using the transformation $u(x, y, z, t)=\exp \left(-r_{v} z\right) v(x, y, z, t), r_{v}=r_{z} / 2 D_{z}$ we can reduce the problem (1) to the problem without a convective term:

$$
\left\{\begin{array}{l}
\frac{\partial v}{\partial t}=\frac{\partial}{\partial x}\left(D_{x} \frac{\partial v}{\partial x}\right)+\frac{\partial}{\partial y}\left(D_{y} \frac{\partial v}{\partial y}\right)+\frac{\partial}{\partial z}\left(D_{z} \frac{\partial v}{\partial z}\right)-D_{z} r_{v}^{2} v \\
x \in\left(0, L_{x}\right), y \in\left(0, L_{y}\right), z \in\left(0, L_{z}\right), t \in\left(0, t_{f}\right) \\
\frac{\partial v(0, y, z, t)}{\partial x}=\frac{\partial v(x, 0, z, t)}{\partial y}=0, \frac{\partial v(x, y, 0, t)}{\partial z}-r_{v} v(x, y, 0, t)=0 \\
D_{x} \frac{\partial v\left(L_{x}, y, z, t\right)}{\partial x}+\alpha_{x}\left(v\left(L_{x}, y, z, t\right)-\exp \left(r_{v} z\right) u_{a x}\right)=0 \\
D_{y} \frac{\partial v\left(x, L_{y}, z, t\right)}{\partial y}+\alpha_{y}\left(v\left(x, L_{y}, z, t\right)-\exp \left(r_{v} z\right) u_{a y}\right)=0 \\
D_{z} \frac{\partial v\left(x, y, L_{z}, t\right)}{\partial z}-0.5 r_{z} v\left(x, y, L_{z}, t\right)+\alpha_{z}\left(v\left(x, y, L_{z}, t\right)-u_{a z} \exp \left(r_{v} L_{z}\right)\right)=0 \\
v(x, y, z, 0)=u 0(x, y, z) \exp \left(r_{v} z\right)
\end{array}\right.
$$

\section{The CAM with the exponential and hyperbolic type integral spline for 1-D initial boundary- value problem}

For motivation of the transformation method we especially consider the 1-D IBV problem with convection: 


$$
\left\{\begin{array}{l}
\frac{\partial u}{\partial t}=\frac{\partial}{\partial z}\left(D_{z} \frac{\partial u}{\partial z}\right)+r \frac{\partial u}{\partial z}+F, z \in\left(0, L_{z}\right), t \in\left(0, t_{f}\right), \\
D_{z} \frac{\partial u(0, t)}{\partial z}-\beta(u(0, t)-u 0)=0, \\
D_{z} \frac{\partial u\left(L_{z}, t\right)}{\partial z}+\alpha\left(u\left(L_{z}, t\right)-u L\right)=0, u(z, 0)=0,
\end{array}\right.
$$

where $\quad F$ - fixed constant, ${ }^{\circ} \mathrm{C} \cdot \mathrm{s}^{-1}$;

$r$ - fixed constant, $\mathrm{m} \cdot \mathrm{s}^{-1}$.

We are looking at the convection term of the PDV in only one $z$-direction to more conveniently show how after transformation a new PDV that does not contain a convective term can be transitioned and the dimension can be further reduced by using a hyperbolic spline with a known parameter. If initially the PDV contains also convective terms in other directions (for example, $x, y$ ), then the process of dimensional reduction can be analogously continued by obtaining ordinary differential equations.

It should be noted that there are enough processes in nature, where the dominant mass or temperature transfer takes place only in the direction of one coordinate, such as $z$. Therefore, physical processes such as filtration, calculation of contamination in underground waters, as well as certain types of combustion processes and other processes that are subject to the given mathematical model may also be modelled through the problem under consideration.

We use without transformation the following exponential type spline approximation:

$$
u(z, t)=u_{z}(t)+m_{z}(t)\left(z-L_{z} / 2\right)+e_{z}(t)\left(\exp \left(a_{z}\left(z-L_{z} / 2\right)\right)-q\right),
$$

where $q=\left(2 / a_{z} L_{z}\right) \sin \left(a_{z} L_{z} / 2\right), u_{z}(t)=\left(L_{z}\right)^{-1} \int_{0}^{L_{z}} u(z, t) d z$ - averaged value.

The unknown functions $m_{z}, e_{z}$ are determined from boundary conditions by $z=0, z=L_{z}$ :

$$
\begin{aligned}
& m_{z}+e_{z} b_{m}-\beta_{1}\left(u_{z}-0.5 m_{z} L_{z}+e_{z}\left(\exp \left(-a_{z} L_{z} / 2\right)-q\right)-u 0\right)=0, \\
& m_{z}+e_{z} b_{m p}+\alpha_{1}\left(u_{z}+0.5 m_{z} L_{z}+e_{z}\left(\exp \left(a_{z} L_{z} / 2\right)-q\right)-u L\right)=0,
\end{aligned}
$$

where $\quad \beta_{1}=\beta / D_{z}, \alpha_{1}=\alpha / D_{z}, b_{m}=a_{z} \exp \left(-a_{z} L_{z} / 2\right), b_{p}=a_{z} \exp \left(a_{z} L_{z} / 2\right), a_{z}=-r / D_{z}$.

Therefore

$$
\begin{gathered}
e_{z}=a_{2} u_{z}+b_{2} ; m_{z}=a_{1} u_{z}+b_{1}, a_{1}=\left(\beta_{1} a_{22}+\alpha_{1} a_{12}\right) / \operatorname{det}, a_{2}=-\left(\beta_{1} a_{21}+\alpha_{1} a_{11}\right) / \operatorname{det}, \\
b_{1}=-\left(\beta_{1} a_{22} u 0+\alpha_{1} a_{12} u L\right) / \operatorname{det}, b_{2}=\left(\beta_{1} a_{21} u 0+\alpha_{1} a_{11} u L\right) / \operatorname{det} ; \operatorname{det}=a_{11} a_{22}-a_{12} a_{21}, \\
a_{11}=1+0.5 \beta_{1} L_{z}, a_{21}=1+0.5 \alpha_{1} L_{z}, a_{12}=b_{m}-\beta_{1}\left(\exp \left(-a_{z} L_{z} / 2\right)-q\right) ; \\
a_{22}=b_{p}+\alpha_{1}\left(\exp \left(a_{z} L_{z} / 2\right)-q\right) .
\end{gathered}
$$
form:

Using the integral averaging from equation (3), we have the initial value problem in the following

$$
\left\{\begin{array}{l}
\frac{\partial u_{z}(t)}{\partial t}=a_{z} e_{z}(t) q\left(D_{z} a_{z}+r\right)+m_{z}(t) r+F, t \in\left(0, t_{f}\right), \\
e_{z}(t)=a_{2} u_{z}(t)+b_{1}, m_{z}(t)=a_{1} u_{z}(t)+b_{1}, u_{z}(0)=0, t \in\left(0, t_{f}\right) .
\end{array}\right.
$$

We have the following solution of these ODEs

$$
u_{z}(t)=\frac{B_{0}}{A_{0}}\left(\exp \left(A_{0} t\right)-1\right)
$$

where $A_{0}=a_{z} a_{2} q\left(D_{z} a_{z}+r\right)+r a_{1}, B_{0}=a_{z} b_{2} q\left(D_{z} a_{z}+r\right)+r b_{1}$.

The 1-D stationary convection-diffusion boundary-value problem (BVP) in the multi-layered plane domain in $z$-direction with different convection velocity in every layer is considered in [11]. In this 
case, the solution to the BVP is discontinuous and can easily be obtained with the conservative averaging method, using the exponential type spline function.

Using the transformation

$$
u(z, t)=\exp \left(-r_{v} z\right) v(z, t), r_{v}=r / 2 D_{z},
$$

we can reduce the problem (3) to the problem without the convective term:

$$
\left\{\begin{array}{l}
\frac{\partial v}{\partial t}=\frac{\partial}{\partial z}\left(D_{z} \frac{\partial v}{\partial z}\right)-D_{z} r_{v}^{2} v+F \exp \left(r_{v} z\right), z \in\left(0, L_{z}\right), t \in\left(0, t_{f}\right), \\
\frac{\partial v(0, t)}{\partial z}-r_{v} v(0, t)-\beta_{1}(v(0, t)-u 0)=0 \\
\frac{\partial v\left(L_{z}, t\right)}{\partial z}-r_{v} v\left(L_{z}, t\right)+\alpha_{1}\left(v\left(L_{z}, t\right)-u L \exp \left(r_{v} L_{z}\right)\right)=0, v(z, 0)=0 .
\end{array}\right.
$$

We use the following hyperbolic type spline approximation:

$$
u(z, t)=v_{z}(t)+m_{z}(t) f_{z 1}+e_{z}(t) f_{z 2},
$$

with the following two fixed hyperbolic functions and the parameter $a_{z}=\left|r_{v}\right|$ :

$$
f_{z 1}=\frac{0.5 L_{z} \sinh \left(a_{z}\left(z-0.5 L_{z}\right)\right)}{\sinh \left(0.5 a_{z} L_{z}\right)}, f_{z 2}=\frac{\cosh \left(a_{z}\left(z-0.5 L_{z}\right)\right)-A_{0 z}}{8 \sinh ^{2}\left(0.25 a_{z} L_{z}\right)},
$$

where $A_{0 z}=\frac{\sinh \left(0.5 a_{z} L_{z}\right)}{0.5 a_{z} L_{z}}, v_{z}(t)=\frac{1}{L_{z}} \int_{0}^{L_{z}} v(z, t) d z$ is the averaged value.

It can be seen, if parameters tend to zero, then in the limit case we get the integral parabolic spline from A. Buikis [12].

The unknown functions $m_{z}, e_{z}$ are determined from the boundary conditions of (5) by $z=0, z=L_{z}$ :

$$
\begin{gathered}
d_{z} m_{z}-k_{z} e_{z}-\left(\beta_{1}+r_{v}\right)\left(v_{z}-0.5 m_{z} L_{z}+e_{z} b_{z}\right)+\beta_{1} u 0=0, \\
d_{z} m_{z}+k_{z} e_{z}-\left(\alpha_{1}-r_{v}\right)\left(v_{z}+0.5 m_{z} L_{z}+e_{z} b_{z}\right)-\alpha_{1} u L \exp \left(r_{v} L_{z}\right)=0, \\
d_{z}=0.5 a_{z} L_{z} \operatorname{coth}\left(0.5 a_{z} L_{z}\right), k_{z}=0.25 a_{z} \operatorname{coth}\left(0.25 a_{z} L_{z}\right), b_{z}=\frac{\cosh \left(0.5 a_{z} L_{z}\right)-A_{0 z}}{8 \sinh ^{2}\left(0.25 a_{z} L_{z}\right)} .
\end{gathered}
$$

Therefore,

$$
\begin{gathered}
e_{z}=a_{2} u_{z}+b_{2}, m_{z}=a_{1} u_{z}+b_{1}, a_{1}=\left(\left(\beta_{1}+r_{v}\right) a_{22}+\left(\alpha_{1}-r_{v}\right) a_{12}\right) / \operatorname{det}, \\
a_{2}=-\left(\left(\beta_{1}+r_{v}\right) a_{21}+\left(\alpha_{1}-r_{v}\right) a_{11}\right) / \operatorname{det}, b_{1}=-\left(\beta_{1} a_{22} u 0+\alpha_{1} a_{12} u L \exp \left(r_{v} L_{z}\right)\right) / \operatorname{det}, \\
b_{2}=\left(\beta_{1} a_{21} u 0+\alpha_{1} a_{11} u L \exp \left(r_{v} L_{z}\right)\right) / \operatorname{det}, \operatorname{det}=a_{11} a_{22}-a_{12} a_{21}, a_{11}=d_{z}+0.5\left(\beta_{1}+r_{v}\right) L_{z}, \\
a_{21}=d_{z}+0.5\left(\alpha_{1}-r_{v}\right) L_{z}, a_{12}=-k_{z}-\left(\beta_{1}+r_{v}\right) b_{z}, a_{22}=k_{z}+\left(\alpha_{1}-r_{v}\right) b_{z} .
\end{gathered}
$$

Using the integral averaging from equation (5), we have the initial value problem in the following form

$$
\left\{\begin{array}{l}
\frac{\partial v_{z}(t)}{\partial t}=D_{z}\left(2 k_{z} e_{z}(t) / L_{z}-r_{v}^{2} v_{z}(t)\right)+F\left(\exp \left(r_{v} L_{z}\right)-1\right) /\left(L_{z} r_{v}\right), \\
e_{z}(t)=a_{2} v_{z}(t)+b_{2}, m_{z}(t)=a_{1} v_{z}(t)+b_{1}, v_{z}(0)=0, t \in\left(0, t_{f}\right) .
\end{array}\right.
$$

We have the following solution of these ODEs

$$
v_{z}(t)=\frac{B_{0}}{A_{0}}\left(\exp \left(A_{0} t\right)-1\right)
$$


where $A_{0}=D_{z}\left(2 k_{z} a_{2} / L_{z}-r_{v}^{2}\right), B_{0}=D_{z} 2 k_{z} b_{2} / L_{z}+F\left(\exp \left(r_{v} L_{z}\right)-1\right) /\left(L_{z} r_{v}\right)$.

\subsection{Some numerical results}

The BVP (3) was solved by two methods. The first method resulted in obtaining a solution $u(z, t)=U$ using exponential type splines. When using transformation $u(z, t)=\exp \left(-r_{v} z\right) v(z, t)$, the BVP (3) was transformed into a BVP (5), its solution $v(z, t)=V_{s}$ was obtained by hyperbolic type splines. The solution of the BVP (3) by the second method was obtained by multiplying $v(z, t)=V_{s}$ by $\exp \left(-r_{v} z\right)$, i.e., $U_{s}=\exp \left(-r_{v} z\right) v(z, t)$.

Problems (3) and (5) were solved with such dimensionless parameters (their numerical values are chosen to solve practical problems), with the aim of clarifying a numerical experiment whether the solution (3) obtained by the first method and the second method does not differ significantly, that is, or the application of the transformation does not reduce the accuracy of the calculations to be carried out:

$$
\begin{gathered}
u 0=1, u L=10, L_{z}=1, \alpha=100, \beta=200, D_{z}=10^{-2}, \\
r=-0.01, F=2, t_{f}=4, t_{j}=0.4 j, j=\overline{0,10}, \\
z_{i}=\frac{1}{40} i, i=\overline{0,40}, a_{z}=0.1 ; 0.05 .
\end{gathered}
$$

The maximal values of the obtained solutions are given for comparison: $u(z, t)=U=10.0032$ (Fig. 1), $v(z, t)=V_{s}=9.534$ (Fig. 2.), $U_{s}=\exp \left(-r_{v} z\right) v(z, t)=10.0053$.

When we solve the BVP (3) through transformation, the accuracy of the calculations is virtually not lost because the maximal error between $U$ and $U_{s}$ is 0.0403 .

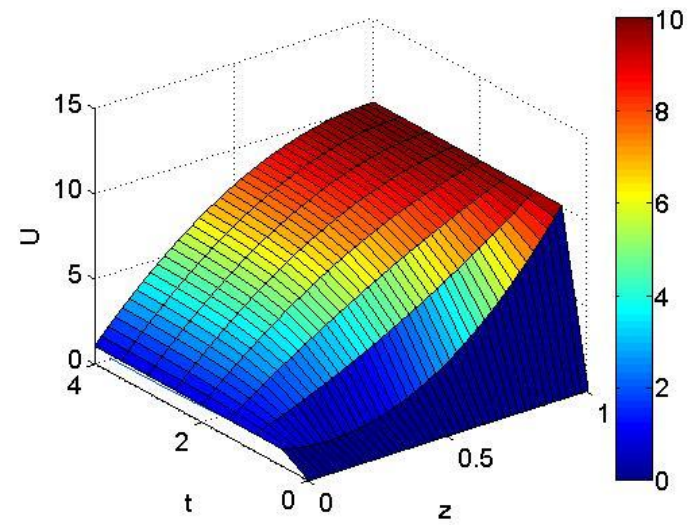

Fig. 1. Exponential type spline solution the surface $u(z, t)=U$

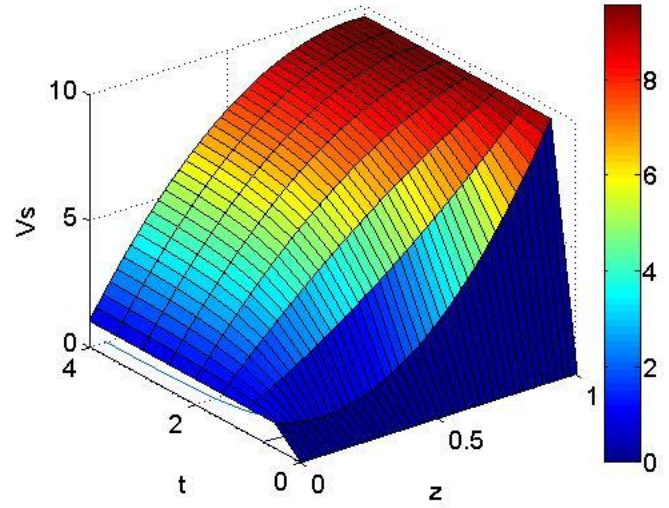

Fig. 2. Hyperbolic type spline solution the surface $v(z, t)=V_{s}$

These numerical calculations in the present paper were performed to demonstrate the usefulness of the transformation by determining the parameter $a_{z}$ of the hyperbolic type spline function. For practical calculations, this method could be used in the work [22], because a similar problem is considered there - the diffusion and convection filtration problem of one substance through the pores of a porous material, which may absorb and immobilize some of the diffusing substance.

The domain consists of a porous material, where incompressible liquid pollutants move in $z$-direction through the pores of a filter. The optimal parameters for the hyperbolic type spline function in $z$-direction without transformation can be obtained only with the method of iterations.

\section{The CAM with the hyperbolic type integral spline approximation for solving the initial boundary-value 3-D problem}

\subsection{The CAM in $z$-direction}


For solving the boundary value problem (2) for every $t>0$ we consider the following hyperbolic type spline approximation with respect to z-direction:

$$
u(x, y, z, t)=v_{z}(x, y, t)+m_{z}(x, y, t) f_{z 1}+e_{z}(x, y, t) f_{z 2},
$$

with the following two fixed hyperbolic type functions

$$
f_{z 1}, f_{z 2}, v_{z}(x, y, t)=\frac{1}{L_{z}} \int_{0}^{L_{z}} v(x, y, z, t) d z
$$

is the averaged value and $a_{z}=r_{v} a_{z}=r_{v}$ is the fixed parameter.

The unknown functions $m_{z}, e_{z}$ are determined from the boundary conditions of (2) by $z=0, z=L_{z}$ :

$$
\begin{gathered}
d_{z} m_{z}-k_{z} e_{z}-r_{v}\left(v_{z}-0.5 m_{z} L_{z}+e_{z} b_{z}\right)+\beta_{1} u 0=0, m_{z}=p_{z} e_{z}+a_{1} v_{z}, \\
p_{z}=\left(k_{z}+r_{v} b_{z}\right) /\left(d_{z}+0.5 L_{z} r_{v}\right), \\
d_{z}=0.5 a_{z} L_{z} \operatorname{coth}\left(0.5 a_{z} L_{z}\right), k_{z}=0.25 a_{z} \operatorname{coth}\left(0.25 a_{z} L_{z}\right), a_{1}=r_{v} /\left(d_{z}+0.5 r_{v} L_{z}\right) \\
D_{z}\left(d_{z} m_{z}+k_{z} e_{z}\right)+a_{2}\left(c_{z}+0.5 m_{z} L_{z}+e_{z} b_{z}\right)-\alpha_{z} u_{a z} \exp \left(r_{v} L_{z}\right)=0,
\end{gathered}
$$

where $\quad b_{z}=\frac{\cosh \left(0.5 a_{z} L_{z}\right)-A_{0 z}}{8 \sinh ^{2}\left(0.25 a_{z} L_{z}\right)}, a_{2}=\alpha_{z}-0.5 r_{z}$.

Therefore,

$$
\begin{gathered}
e_{z}=\left(g_{z}\right)^{-1}\left(u_{a z}-a_{6} v_{z}\right), g_{z}=\left(a_{3} p_{z}+a_{4}\right) / a_{5}, a_{6}=\left(a_{2}+a_{1} a_{3}\right) / a_{5}, e_{z}=\left(u_{a z}-a_{6} v_{z}\right) / g_{z}, \\
g_{z}=\left(a_{3} p_{z}+a_{4}\right) / a_{5}, a_{6}=\left(a_{2}+a_{1} a_{3}\right) / a_{5}, a_{2}=\alpha_{z}-0.5 r_{z}, a_{3}=D_{z} d_{z}+0.5 a_{2}, \\
a_{4}=D_{z} k_{z}+a_{2} b_{z}, a_{5}=\alpha_{z} \exp \left(r_{v} L_{z}\right), m_{z}=p_{z} e_{z}+a_{1} v_{z} .
\end{gathered}
$$

Now the initial-boundary value 2-D problem is in following form

$$
\left\{\begin{array}{l}
\frac{\partial v_{z}}{\partial t}=\frac{\partial}{\partial x}\left(D_{x} \frac{\partial v_{z}}{\partial x}\right)+\frac{\partial}{\partial y}\left(D_{y} \frac{\partial v_{z} u}{\partial y}\right)+a_{0 z}^{2}\left(u_{a z}-a_{6} v_{z}\right)-D_{z} r_{v}^{2} v_{z} \\
\frac{\partial v_{z}(0, y, t)}{\partial x}=\frac{\partial v_{z}(x, 0, t)}{\partial y}=0 \\
D_{x} \frac{\partial v_{z}\left(L_{x}, y, t\right)}{\partial x}+\alpha_{x}\left(v_{z}\left(L_{x}, y, t\right)-C_{v} u_{a x}\right)=0, \\
D_{y} \frac{\partial v_{z}\left(x, L_{y}, t\right)}{\partial y}+\alpha_{y}\left(v_{z}\left(x, L_{y}, t\right)-C_{v} u_{a y}\right)=0, \\
v_{z}(x, y, 0)=C_{v} u 0(x, y), x \in\left(0, L_{x}\right), y \in\left(0, L_{y}\right), t \in\left(0, t_{f}\right),
\end{array}\right.
$$

where $a_{0 z}^{2}=\left(2 D_{z} k_{z}\right) / L_{z} g_{z}, C_{v}=\left(L_{z}\right)^{-1} \int_{0}^{L_{z}} \exp \left(r_{v}, z\right) d z=\left(\exp \left(r_{v}, L_{z}\right)-1\right) / r_{v}, L_{z}$.

\subsection{The CAM in y-direction}

Using the averaged method with respect to $y$ we use

$$
v_{y}(x, t)=\left(L_{y}\right)^{-1} \int_{0}^{L_{y}} v_{z}(x, y, t) d y .
$$

For the following hyperbolic type spline approximation

$$
v_{z}(x, y, t)=v_{y}(x, t)+m_{y}(x, t) f_{y 1}+e_{y}(x, t) f_{y 2},
$$

we have 


$$
f_{y 1}=\frac{0.5 L_{y} \sinh \left(a_{y}\left(y-0.5 L_{y}\right)\right)}{\sinh \left(0.5 a_{y} L_{y}\right)}, f_{y 2}=\frac{\cosh \left(a_{y}\left(y-0.5 L_{y}\right)\right)-A_{0 y}}{8 \sinh ^{2}\left(0.25 a_{y} L_{y}\right)},
$$

where $A_{0 y}=\frac{\sinh \left(0.5 a_{y} L_{y}\right)}{0.5 a_{y} L_{y}}$,

and as the parameter we choose

$$
\begin{aligned}
& a_{y}=a_{0 z} \sqrt{a_{6} / D_{y}}+r_{v} \sqrt{D_{z} / D_{y}} \\
& a_{y}=a_{0 z} \sqrt{a_{6} / D_{y}}+r_{v} \sqrt{D_{z} / D_{y}} .
\end{aligned}
$$

Similarly, we determine the unknown functions $m_{z}, e_{z}$ from the boundary conditions by $z=0, z=L_{z}$ :

$$
\begin{gathered}
e_{y}=\left(u_{a y} C_{v}-v_{y}\right) / g_{y}, g_{y}=b_{y}+0.5 p_{y} L_{y}+2 k_{y} D_{y} / \alpha_{y}, m_{y}=p_{y} e_{y}, p_{y}=k_{y} / d_{y}, \\
d_{y}=0.5 a_{y} L_{y} \operatorname{coth}\left(0.5 a_{y} L_{y}\right), k_{y}=0.25 a_{y} \operatorname{coth}\left(0.25 a_{y} L_{y}\right), b_{y}=\frac{\cosh \left(0.5 a_{y} L_{y}\right)-A_{0 y}}{8 \sinh ^{2}\left(0.25 a_{y} L_{y}\right)} .
\end{gathered}
$$

The initial-boundary value 1-D problem is in following form

$$
\left\{\begin{array}{l}
\frac{\partial v_{y}}{\partial t}=\frac{\partial}{\partial x}\left(D_{x} \frac{\partial v_{y}}{\partial x}\right)+a_{0 y}^{2}\left(u_{a y}-v_{y}\right)+a_{0 z}^{2}\left(u_{a z}-a_{6} v_{y}\right)-D_{z} r_{v}^{2} v_{y}=0, \\
\frac{\partial v_{y}(0, t)}{\partial x}=0, D_{x} \frac{\partial v_{y}\left(L_{x}, t\right)}{\partial x}+\alpha_{x}\left(v_{y}\left(L_{x}, t\right)-C_{v} u_{a x}\right)=0, \\
v_{y}(x, 0)=C_{v} u 0(x), x \in\left(0, L_{x}\right), t \in\left(0, t_{f}\right),
\end{array}\right.
$$

where $a_{0 y}^{2}=\left(2 D_{y} k_{y}\right) / L_{y} g_{y}, c_{o y}(x)=\left(L_{y}\right)^{-1} \int_{0}^{L_{y}} c_{o z}(x, y) d y$.

\subsection{The CAM in $x$-direction}

It is possible to proceed averaging also in $x$-direction

$$
v_{x}(t)=\left(L_{x}\right)^{-1} \int_{0}^{L_{x}} v_{y}(x, t) d x .
$$

For the following hyperbolic type spline approximation

$$
v_{y}(x, t)=v_{x}(t)+m_{x}(t) f_{x 1}+e_{x}(t) f_{x 2},
$$

we have

$$
f_{x 1}=\frac{0.5 L_{x} \sinh \left(a_{x}\left(x-0.5 L_{x}\right)\right)}{\sinh \left(0.5 a_{x} L_{x}\right)}, f_{x 2}=\frac{\cosh \left(a_{x}\left(x-0.5 L_{x}\right)\right)-A_{0 x}}{8 \sinh ^{2}\left(0.25 a_{x} L_{x}\right)},
$$

where $A_{0 x}=\frac{\sinh \left(0.5 a_{x} L_{x}\right)}{0.5 a_{x} L_{x}}$,

and as the parameter we choose

$$
a_{x}=\sqrt{\left(a_{0 z}^{2} a_{6}+a_{0 y}^{2}+D_{z} r_{v}^{2}\right) / D_{x}}
$$

Similarly, we determine the unknown functions $m_{x}, e_{x}$ from the boundary conditions by $x=0$, $x=L_{x}$ and

$$
e_{y}=\left(u_{a x} C_{v}-v_{x}\right) / g_{x}, g_{x}=b_{x}+0.5 p_{x} L_{x}+2 k_{x} D_{x} / \alpha_{x}, m_{x}=p_{x} e_{x}, p_{x}=k_{x} / d_{x},
$$




$$
d_{x}=0.5 a_{x} L_{x} \operatorname{coth}\left(0.5 a_{x} L_{x}\right), k_{x}=0.25 a_{x} \operatorname{coth}\left(0.25 a_{x} L_{x}\right), b_{x}=\frac{\cosh \left(0.5 a_{x} L_{x}\right)-A_{0 x}}{8 \sinh ^{2}\left(0.25 a_{x} L_{x}\right)} .
$$

From problem (8) the initial problem of linear ODEs follows

$$
\left\{\begin{array}{l}
\frac{\partial c_{x}(t)}{\partial t}=a_{0 y}^{2}\left(u_{a y}-v_{x}(t)\right)+a_{0 z}^{2}\left(u_{a z}-a_{6} v_{x}(t)\right)-D_{z} r_{v}^{2} v_{x}(t)+a_{0 x}^{2}\left(u_{a x}-v_{x}(t)\right)=0, \\
v_{x}(0)=C_{v} u 0, t \in\left(0, t_{f}\right),
\end{array}\right.
$$

where $a_{0 x}^{2}=\left(2 D_{x} k_{x}\right) / L_{x} g_{x}$.

The solution of this problem can be obtained with the classical methods.

For $u 0=0$ we have,

$$
c_{x}(t)=\left(A_{0} / B_{0}\right)\left(1-\exp \left(-B_{0} t\right)\right)
$$

where $\quad A_{0}=a_{0 y}^{2} u_{a y}+a_{0 z}^{2} u_{a z}+a_{0 x}^{2} u_{a x}, B_{0}=a_{0 y}^{2}+a_{6} a_{0 z}^{2}+a_{0 x}^{2}+D_{z} r_{v}^{2}$.

In the stationary case we have

$$
c_{x}=A_{0} / B_{0} .
$$

For fixed $t_{f}$ we have:

$$
\begin{gathered}
v_{y}\left(x, t_{f}\right)=v_{x}\left(t_{f}\right)+m_{x}\left(t_{f}\right) f_{x 1}+e_{x}\left(t_{f}\right) f_{x 2}, e_{x}\left(t_{f}\right)=\left(u_{a x}-v_{x}\left(t_{f}\right)\right) / g_{x}, m_{x}\left(t_{f}\right)=p_{x} e_{x}\left(t_{f}\right), \\
v_{z}\left(x, y, t_{f}\right)=v_{y}\left(x, t_{f}\right)+m_{y}\left(x, t_{f}\right) f_{y 1}+e_{y}\left(x, t_{f}\right) f_{y 2}, e_{y}\left(x, t_{f}\right)=\left(u_{a y}-v_{y}\left(x, t_{f}\right)\right) / g_{y} . \\
m_{y}\left(x, t_{f}\right)=p_{y} e_{y}\left(x, t_{f}\right), v\left(x, y, z, t_{f}\right)=v_{z}\left(x, y, t_{f}\right)+m_{z}\left(x, y, t_{f}\right) f_{z 1}+e_{z}\left(x, y, t_{f}\right) f_{z 2}, \\
e_{z}\left(x, y, t_{f}\right)=\left(u_{a z}-a_{6} v_{z}\left(x, y, t_{f}\right)\right) / g_{z}, m_{z}\left(x, y, t_{f}\right)=p_{z} e_{z}\left(x, y, t_{f}\right)+a_{1} v_{z}\left(x, y, t_{f}\right), \\
u\left(x, y, z, t_{f}\right)=\exp \left(-r_{v} z\right) v\left(x, y, z, t_{f}\right) .
\end{gathered}
$$

\section{Numerical approximations with ADI method for solving the initial boundary-value 3-D problem}

We use uniform grid in the space $((K+1) \times(\tilde{N}+1) \times(M+1))$ :

$$
\begin{gathered}
\left\{\left(z_{k}, y_{i}, x_{j}\right), z_{k}=(k-1) \cdot h_{z}, y_{i}=(i-1) \cdot h_{y}, y_{i}=(i-1) \cdot h_{y}, x_{j}=(j-1) \cdot h_{x}, i=\overline{1, \tilde{N}+1},\right. \\
\left.j=\overline{1, M+1}, k=\overline{1, K+1}, K \cdot h_{z}=L_{z}, \tilde{N} \cdot h_{y}=L_{y}, M \cdot h_{x}=L_{x}\right\} .
\end{gathered}
$$

For the time $t$ we use the moments $t_{n}=n \tau, n-0,1, \ldots$, subscripts $(k, i, j, n)$ refer to $z, y, x, t$ indices with the mesh spacing and for approximation of the function $u(z, y, x, t)$ we have the grid function with values

$$
U_{k, i, j}^{n} \approx u\left(z_{k}, y_{i}, x_{j}, t_{n}\right) .
$$

For solving the 3-D problem (1) we use the discrete approximation

$$
\left(U_{k, i, j}^{n+1}-U_{k, i, j}^{n}\right) / \tau=\left(\Lambda_{z}+\Lambda_{y}+\Lambda_{x}\right) U_{k, i, j}^{n+1}, n \geq 0, k=\overline{1, K+1}, i=\overline{1, \tilde{N}+1}, j=\overline{1, M+1}
$$

And ADI method by J. Douglas and H. H. Rachford [19]:

$$
\left\{\begin{array}{l}
\left(U_{k, i, j}^{n+1 / 3}-U_{k, i, j}^{n}\right) / \tau=\Lambda_{z} U_{k, i, j}^{n+1 / 3}+\Lambda_{y} U_{k, i, j}^{n}+\Lambda_{x} U_{i, j}^{n}+f_{i, j}^{n}, k=\overline{2, K}, i=\overline{2, N}, j=\overline{2, M}, \\
\left(U_{k, i, j}^{n+2 / 3}-U_{k, i, j}^{n+1 / 3}\right) / \tau=\Lambda_{y}\left(U_{k, i, j}^{n+2 / 3}-U_{k, i, j}^{n}\right), k=\overline{1, K+1}, i=\overline{2, N}, j=\overline{1, M+1} \\
\left(U_{k, i, j}^{n+1}-U_{k, i, j}^{n+2 / 3}\right) / \tau=\Lambda_{x}\left(U_{k, i, j}^{n+1}-U_{k, i, j}^{n}\right), k=\overline{1, K+1}, i=\overline{1, \tilde{N}+1}, j=\overline{2, M}
\end{array}\right.
$$


After eliminating the fractional time moments $t_{n+1 / 3}, t_{n+2 / 3}$, we obtain the previous discrete problem with the approximation error $O\left(\tau^{2}\right)$. Here $\Lambda_{x}, \Lambda_{y}, \Lambda_{z}$ are the discrete difference operators, approximated expressions

$$
\frac{\partial}{\partial x}\left(D_{x} \frac{\partial u(x, y, z, t)}{\partial x}\right), \frac{\partial}{\partial y}\left(D_{y} \frac{\partial u(x, y, z, t)}{\partial y}\right), \frac{\partial}{\partial z}\left(D_{z} \frac{\partial u(x, y, z, t)}{\partial z}\right)+r_{z} \frac{\partial u(x, y, z, t)}{\partial z}
$$

with respect to $x, y, z$ and boundary conditions with central differences.

For solving $U^{n+1 / 3}, U^{n+2 / 3}$ and $U^{n+1}$ we use Thomas algorithm in $z, y$ and $x$ directions, respectively.

\section{Results and discussion}

This chapter checks the accuracy of the calculations with the CAM, using the ADI method.

Two solutions to the 3-D problem (1) are compared, one of which was obtained with the CAM using the above mentioned transformation, while the second solution was obtained with the ADI method (see Chapter 4).

The numerical results are obtained for the number of the grid points for $(x, y, z),(\tilde{N}=M=21$, $K=19)$ and for the following dimensionless parameters of BVP (1):

$$
\begin{gathered}
L_{z}=1, L_{y}=2, L_{x}=3, \alpha_{z}=200, \alpha_{x}=\alpha_{y}=20, c_{a z}=1, c_{a x}=3, c_{a y}=2, \\
D_{y}=0.3 \cdot 10^{-2}, D_{x}=0.3 \cdot 10^{-2}, D_{z}=10^{-2}, r_{z}=0.1 .
\end{gathered}
$$

When using the ADI method, the stationary averaged solution $u_{z}\left(x, y, z, t_{f}\right)$ was chosen as the initial condition. During proceeding of the ADI method, a stationary solution was obtained with $\tau=1, t_{f}=300$ and with the maximal error (difference between two adjacent iterations) $10^{-8}$.

For comparison of both of the presented methods the maximal values of the obtained solutions are the following: $u\left(x, y, 0, t_{f}\right)=2.9956$ for averaged method (Fig. 3) and $u\left(x, y, 0, t_{f}\right)=2.9964$ for ADI method (Fig. 4). Depending on the number of the grid points, $(N, M, K)$ we have following maximal values for the ADI method: $2.9964(\tilde{N}=M=21, K=19), 2.9963(\tilde{N}=M=31, K=29)$.

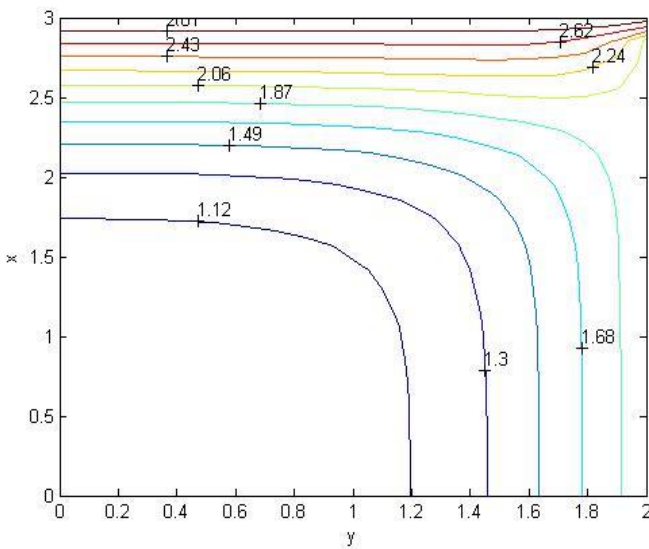

Fig. 3. Levels of averaged solution $u\left(x, y, 0, t_{f}\right)$

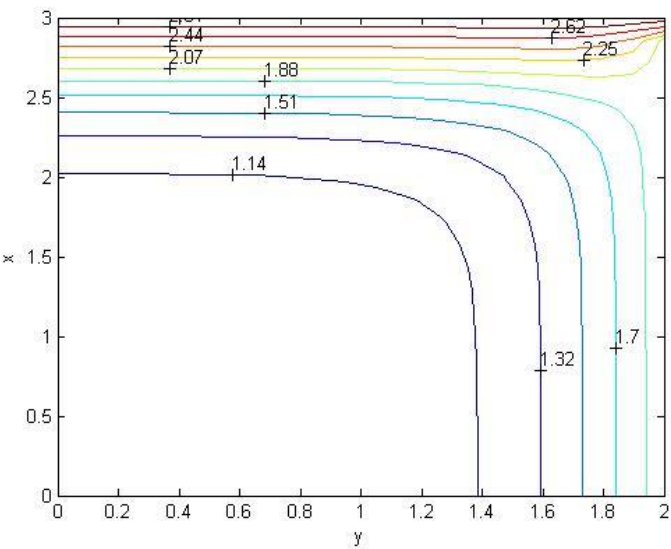

Fig. 4. Levels of numerical solution $u\left(x, y, 0, t_{f}\right)$

It appears that the CAM, in terms of precision, is perfectly similar to the ADI method, which requires both an adequate theoretical knowledge base on the method itself and adequate knowledge and skills for numerical execution of its algorithm.

It should also be noted that the usage of the CAM shows the possibility of acquiring new knowledge: the newly designed algorithms take the form of resolving previously studied tasks/problems in another way, which is different from traditional algorithms, and are often even simpler and easier to execute.

\section{Conclusions}


1. The present paper deals with the special transformation created that transforms the 3-D heat transfer boundary-value problem (BVP) with convection in $z$-direction to a corresponding problem (BVP) without convection.

2. Applying the method, the 1-D initial-boundary value problem (IBVP) with convection is solved using the special transformation - thus obtaining the IBVP without a convective term, which allowed to determine the coefficients of the spline function in such a way that the calculation error is minimal.

3. The corresponding 3-D BVP with the special transformation was also modified into a problem without a convective term, and further, based on the CAM, the problem obtained was reduced to 2$\mathrm{D}$ and 1-D problems, using the integral hyperbolic splines with the parameters (the spline function coefficients) found.

4. The stationary numerical solution is compared with the spline solution. The maximal absolute value of the difference between the corresponding numerical and averaged solutions was $0.1-0.2$ percent.

5. The methodology with the transformation described above allows us to solve also the tasks of a wider class - with convection also in y-direction and $\mathrm{x}$-direction, thereby reducing the studying BVP to the problem without convective terms, which, in turn, allows us to determine the spline function coefficients for minimizing the calculation errors.

\section{References}

[1] Favre-Marinet M., Tardu S. Convective heat transfer. Solved problems WILEY, ISTE Ltd, 2009 $373 \mathrm{p}$.

[2] Kalis H., Buikis A., Aboltins A., Kangro I. Special splines of hyperbolic type for the solutions of heat and mass transfer 3-D problems in porous multi-layered axial symmetry domain. Mathematical Modelling and Analysis, vol. 22, issue 4, 2017, pp. 425-440.

[3] Aboltins A., Kalis H., Pulkis K., Skujans J., Kangro I. Mathematical modeling of heat transfer problem for two layered gypsum board products exposed to fire, Proceedings of International conference "16th International Scientific Conference Engineering for Rural Development", May 24-26, 2020, Jelgava, Latvia, vol. 16, 2017, pp. 1369-1376.

[4] Maciejewska B. Piasecka M. An application of the non-continuous Trefftz method to the determination of heat transfer coefficient for flow boiling in a mini channel. Heat Mass Transf. $2017,53,1211-1224$.

[5] Simoes N., Tadeu A. 3D transient heat transfer by conduction and convection across a 2D medium using boundary element model, CMES, vol.9, No3, 2005, pp. 221-233.

[6] Purusothaman A., Murugesan K., Chamkla A.J. 3D modeling of natural convective heat transfer from a varying restangular heat generating source J. of thermal Analysis and Calorimetry 138., 2019, pp. 597-608.

[7] Saglam M., Sarper B., Aydin O. Natural convection in an enclosure with discretely heated sidewall: heatlines and flow visualization. J. of Applied Fluid Mechanics vol.11, No 1, 2018, pp. 271-284.

[8] Pavlenko I., Liaposhchenko O., Ochowiak M., Olszewski R., Demianenko M., Starynskyi O., Ivanov V., Yanovych V., Włodarczak S., Doligalski M. Three_dimensional Mathematical model of the liquid film downflow on vertical surface. Energies 2020, 13, 1938, $15 \mathrm{pp}$.

[9] Saqib M., Hasnain S., Mashat, D. Computational solutions of three dimensional convectiondiffusion equation using fourth order time efficient alternating direction implicit scheme. J. of Materials Science and Mathematical Physics, 085306, 2017, https://doi.org/10.1063/1.4996341

[10] Kangro I., Kalis H., Gedroics A., Teirumnieka E., Teirumnieks E. On mathematical modelling of metals distribution in peat layers. Mathematical Modelling and Analysis, vol. 19, issue 4, 2014, pp. 568-588.

[11] Aboltins A., Kalis H., Kangro I. Conservative averaging method for solving some nonlinear heat transfer problems related to combustion. Proceedings of International conference "19th International Scientific Conference Engineering for Rural Development”, May 20-22, 2020, Jelgava, Latvia, vol. 19, 2020, pp. 1698-1705.

[12] Buikis A. The analysis of schemes for modelling some processes of filtration in the underground. Acta Universitatis Latviensis, Riga, vol. 592, 1994, pp. 135-138 (in Latvian). 
[13] Buikis A., Kalis H., Kangro I. Special splines of exponential type for the solutions of mass transfer problems in multi-layer domains. Mathematical Modelling and Analysis, vol. 21, issue 4, 2016, pp. 450-465.

[14] Buikis A., Kalis H., Kangro I. Special hyperbolic type spline for mass transfer problems in multilayer 3-D domains. Mathematical and Computational Methods in Applied Sciences. Proceedings of International conference" 3-rd International Scientific Conference on Applied, Numerical and Computational Mathematics", August 17-19, 2015, Sliema, Malta, pp. 25-34.

[15] Kangro I., Kalis H., Teirumnieka E., Teirumnieks E. Special hyperbolic type approximation for solving of 3-D two layer stationary diffusion problem. Proceedings of International conference"12th International Scientific Conference Environment Technology Resources", June 20-22, 2019, Rezekne, Latvia, vol. 3, pp. 95-100.

[16] Vilums R., Buikis, A. A. Transient heat conduction in 3D fuse modelled by conservative averaging method. Topics in advanced theoretical and applied mechanics. Proceedings of International conference"3rd WSEAS International Conference on Applied and Theoretical Mechanics", December 14-16, 2007, Puerto de la Cruz, Spain, pp. 54-63.

[17] Vilums R., Buikis, A. Rudevics, A. Cylindrical model of transient heat conduction in automotive fuse using conservative averaging method. Applied and computational mathematics, $2^{\text {nd }}$ edition. Proceedings of International conference"13th WSEAS International Conference on Applied Mathematics", December 15-17, 2008, Puerto de la Cruz, Spain,_pp. 355-364.

[18]Zhang B., Sorathia, K., Lyon J-G, et al. Conservative averaging-reconstruction techniques (Ring Average) for 3-D finite-volume MHD solvers with axis singularity. Journal of computational physics, vol. 376, 2019, pp. 276-294.

[19] Douglas J., Rachford H. H. On the numerical solution of heat conduction problems in two and three space variables. Trans. Amer. Math. Soc., vol. 82, issue 2, 1956, pp. 421-489.

[20] Cengel, Y.A., Afshin, J.G. Heat and mass transfer: Fundamentals and Applications. 5th Edition. New York: McGraw-Hill, 2015. 850p.

[21] Bergman, T.L., Lavine, A.S. Fundamentals of Heat and mass transfer. 6th Edition. New York: John Wiley \& Sons, 2007. 997p.

[22] Kangro I., Kalis H. On Mathematical Modelling of the Solid-Liquid Mixtures Transport in Porous Axial-Symmetrical Container with Henry and Langmuir Sorption Kinetics. Mathematical Modelling and Analysis, vol. 23, issue 4, 2018, pp. 554-567. 